\title{
The emergence of science-driven entrepreneurship in China: a case study of technological innovation in nano-pigment inks
}

\author{
Yu Meng* \\ Public Policy and Global Affairs Programme, \\ School of Humanities and Social Sciences, \\ Nanyang Technological University, \\ 637332, Singapore \\ and \\ Program in Science, Technology and Innovation Policy, \\ School of Public Policy, \\ Georgia Institute of Technology, \\ Atlanta, GA 30332-0345, USA \\ E-mail: mengyu2211@hotmail.com \\ *Corresponding author

\section{Philip Shapira} \\ Manchester Institute of Innovation Research, \\ Manchester Business School, \\ University of Manchester, \\ Manchester, M13 9PL, UK \\ and \\ Program in Science, Technology and Innovation Policy, \\ School of Public Policy, \\ Georgia Institute of Technology, \\ Atlanta, GA 30332-0345, USA \\ E-mail: pshapira@mbs.ac.uk

\section{Li Tang}

School of Public Economics and Administration,

Shanghai University of Finance and Economics,

Shanghai, 200433, China

and

Program in Science, Technology and Innovation Policy,

School of Public Policy,

Georgia Institute of Technology,

Atlanta, GA 30332-0345, USA

E-mail: Tang.li@shufe.edu.cn

\begin{abstract}
All countries face challenges in commercialising scientific advances in emerging technology domains. As an emerging economy, China is rapidly evolving its innovation system, but still faces many issues in linking scientific
\end{abstract}


development with industrial applications. While research and development (R\&D) investment in Chinese science has increased significantly in recent years, there are substantial lags and barriers in the deployment of new technology knowledge by business, including by small and medium-sized enterprises. Yet, there are important signs of change within research organisations and in the policy support structures for research commercialisation in China. To explore these developments, this study examines a specific case within the domain of nanotechnology, an emerging technology domain where China is now among the world's biggest R\&D performers. We investigate an example in China of science-driven entrepreneurship in a new technology for producing nano-pigment inks for digital inkjet textile printing. The contextual conditions and the development and commercialisation processes of this focal technology are analysed, as we examine the strategies used by the research team to spin-off their research towards commercialisation. We identify key factors in the Chinese innovation system that respectively facilitated and hindered this research commercialisation example, and consider managerial and policy implications raised by the case.

Keywords: nanotechnology, nano-pigment ink, digital printing, textiles, innovation, China.

Reference to this paper should be made as follows: Meng, Y., Shapira, P. and Tang, L. (2013) 'The emergence of science-driven entrepreneurship in China: a case study of technological innovation in nano-pigment inks', Int. J. Entrepreneurship and Innovation Management, Vol. 17, Nos. 1/2/3, pp.162-176.

Biographical notes: Yu Meng is currently a Postdoctoral Fellow in the Public Policy and Global Affairs Program at Nanyang Technological University, Singapore. She received her $\mathrm{PhD}$ in the School of Public Policy at Georgia Institute of Technology, and was a Visiting Assistant Professor in the Department of Science, Technology and Society/Public Policy at Rochester Institute of Technology (Rochester, NY, USA) and a Research Fellow in Fraunhofer Institute for System and Innovation Research (Karlsruhe, Germany). Her research focuses on science, technology, and innovation (STI) policy, in particular the issues in Chinese innovation system and those regarding gender inequalities.

Philip Shapira is Professor of Innovation, Management and Policy at the Manchester Institute of Innovation Research, Manchester Business School, University of Manchester, UK; and Professor of Public Policy at Georgia Institute of Technology, Atlanta, USA. He is associated with the Center for Nanotechnology in Society at Arizona State University and leads a Project on Emerging Technologies and Implications of Next Generation Innovation Systems Development in China and Russia (sponsored by the UK Economic and Social Research Council). He is a co-editor of The Theory and Practice of Innovation Policy: An International Research Handbook (Edward Elgar, 2010).

Li Tang is an Assistant Professor in the School of Public Economics and Administration, Shanghai University of Finance and Economics, Shanghai, China. She holds a doctoral degree in Public Policy from the Georgia Institute of Technology, Atlanta, USA. Her areas of research interest include S\&T policy analysis, innovation-based economic development, research evaluation, and scientific migration. She has co-authored several journal articles which appeared in Journal of Informetrics, Journal of the American Society for Information Science and Technology, Journal of Technology Transfer, and Scientometrics. 


\section{Introduction}

Nanotechnology - the development and assembly at the atomic and molecular scale of advanced materials and devices with novel properties - is expected to be one of the leading drivers of technological and economic development over the next few decades. For much of the twentieth century, innovations in materials have emerged from the USA, Europe, Japan, and other advanced economies, but today China has emerged as a major player in the development of nanotechnology (nano) materials. China is now among the world's top two producers of scientific articles in nanotechnology (alongside the USA) and has seen a strong increase in domestic patenting (Liu and Zhang, 2005; Tang and Shapira, 2011; Youtie et al., 2008; Zhou and Leysdorff, 2006). The conditions for this dramatic growth have been fostered by the Chinese government's proactive engagement in prioritising nanotechnology and investing heavily in national policy instruments, programmes, and centres to encourage indigenous research and development (R\&D) in this field (Shapira and Wang, 2009). Another critical component of this story is the rise of entrepreneurial high technology companies in the nanomaterials domain. This is an important development in an economy traditionally based on large state-affiliated enterprises and, more recently, on private but often low-technology producers of such items as electrical and mechanical components, textiles, and processed materials. While there is growing recognition of the larger picture of China's rise as a scientific super-power in nanotechnology, relatively little is known about how nanotechnology is developed and commercialised in China at the level of individual entrepreneurial ventures.

A noticeable feature of innovation in nanotechnology is its strong basis in science. A significant share of nanotechnology patent applications and patent grants in the USA originate from universities (Mowery, 2011). This pattern of commercialisation is less clearly developed in China, where university-industry relationships are still evolving. There are persistent structural issues in the Chinese innovation system, including the prevalence of top-down information flows, the lack of interaction and trust among different actors, and difficulties in raising private-sector funds for long-term technological development by enterprises. This raises the question of how the commercialisation of nanotechnology in new enterprises is linked to scientific advancement in research institutions and is influenced by the characteristics of the national innovation system. To probe this question at the micro-scale, we examine a case of innovation in the production of nano-pigment inks. Our case study is a nano-related technology developed and commercialised primarily by a university research team. By documenting and analysing this case in detail, we seek to better understand the dynamics underlying science-driven technology development and the evolving innovation system in China.

\section{Method}

Our investigation uses a case study approach. This is "a method for learning about a complex instance, based on a comprehensive understanding of that instance obtained by extensive description and analysis of that instance taken as a whole and in its context" [GAO, (1990), p.14]. As a technique for exploratory and in-depth investigation 
(Yin, 2009; Shepard and Greene, 2003; Babbie, 2000), this approach emphasises the importance of contextual and longitudinal perspectives. In our case, primary information is collected from interviews with relevant informants. We also identify and analyse secondary sources including governmental, organisational and other relevant technical documents.

The process of evidence collection and case investigation proceeded through three phases. In the first phase, secondary data were collected from a wide variety of sources including national and local government websites, university websites, newspapers, scholarly publications, patents, and researchers' online curriculum vitae. The second phase comprised interviews with key informants related to the case. These informants included lead researchers, an officer in the State Intellectual Property Office (SIPO) of China, local government science and technology executives, senior managers of local textile companies, and senior executives in the local textile industry consortium. We employed semi-structured interview protocols (with Institutional Review Board approval). Field work and personal interviews were conducted in December 2009. We promised anonymity and thus use pseudonyms for interviewees and affiliated universities in this publication. In the third phase, based on information obtained from these sources, we organised and analysed the evidence. Furthermore, additional documented information was collected and follow-up telephone calls were made with interviewees for clarification and confirmation.

\section{Context}

\subsection{The focal technology}

The focus of this study is the technology of producing nano-pigment inks for digital inkjet textile printing. It is an advanced technology not only due to the complexity and novelty of manipulating nano-scale pigment particles and making a stable waterborne ink system containing nano-pigments, but also because digital inkjet printing is a new and advanced technique in the textile industry. This section provides a brief overview of digital inkjet textile printing and summarises the advantages of nano-pigment ink technology.

Digital inkjet printing for textiles refers to the process of directly printing designs using computer aided design and specialised dying and printing software systems. New digital printing processes have many advantages over traditional screen printing. Although the global textile printing market is still dominated by screen printing, digital inkjet printing has been growing in scale and sophistication since the early 1980s, bolstered by the rapid development of information and communication technologies and by increasing industry attention to environmental impacts (Cahill, 2006; Ohno, 2006). With direct inkjet sampling, the preparation of colour-separated positives or screens is no longer necessary, which makes the industrial process more simple, flexible, and efficient. This facilitates 'just-in-time' and customisation requirements (see Figure 1). Digital inkjet printing is also more environment-friendly than conventional printing processes. Compared with conventional printing, for the same output of printed textiles, digital printing consumes $30 \%$ less water, uses less dye and reduces water waste and electricity requirements by $97 \%$ and $45 \%$ respectively (Ohno, 2006). Additionally, higher image 
quality is obtained from digital inkjet printing (up to 2,880 dots per inch compared with 200 dots per inch for the conventional printing process).

Figure 1 Comparison of the process of (a) conventional textile printing and (b) digital inkjet printing (see online version for colours)

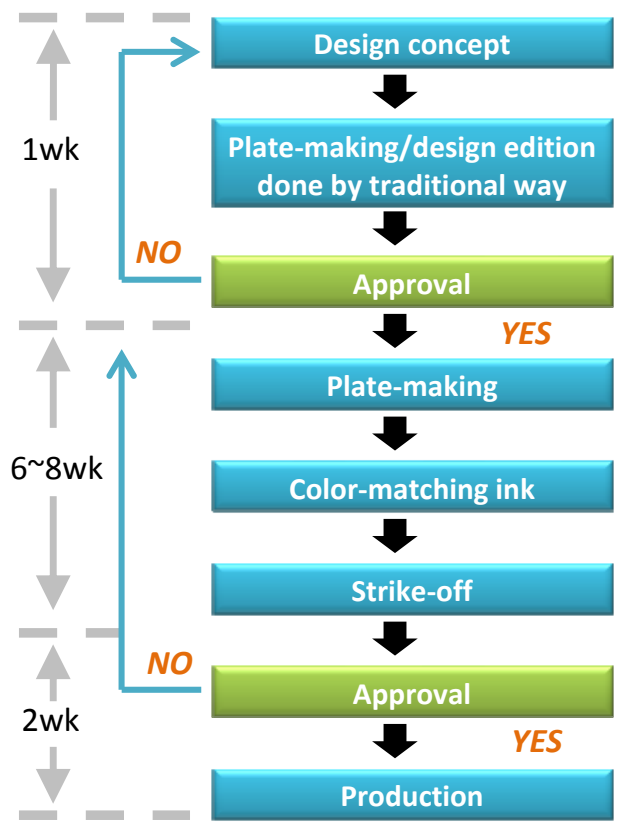

(a)

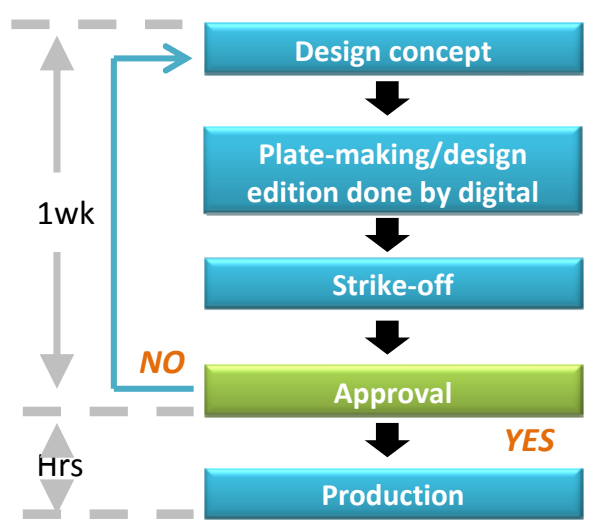

(b)

Source: Ohno (2006)

Ink is itself a crucial component of the digital inkjet printing technology used in the textile industry. The development of inks thus affects (either promotes or limits) the application of the new digital printing processes. Currently, commercial inks for digital inkjet textile printing include dye-based (acid, reactive, and disperse) and pigment-based inks. Scientists and manufacturers have recognised that pigments have many advantages over dyes in textile dyeing and printing, such as offering a wider range of colours, better light fastness, and greater acid stability (Fang, 2006). Pigment-based inks are adaptable to more fabric types and generate fewer environmental issues as they require less pre- and post-treatments (Table 1). However, since pigments are not dissolvable in water or other organic solvents, a series of challenges for full utilisation are presented. The following three challenges are most prominent (Fu, 2006; Fang et al., 2004; Fang, 2006):

- developing an optimal method of preparing ultrafine pigment particles.

- $\quad$ formulating multifunctional agent(s) that can work well as both dispersion agent(s) and binder(s) to prepare a dispersion system containing pigment particles.

- making a nano-pigment dispersion system that has adequate permanence properties, enough shelf lifetime, low viscosity, and sufficient colour density. 
Table 1 Comparisons of inks for digital inkjet textile printing

\begin{tabular}{|c|c|c|c|c|}
\hline Ink type & & Fabrics & Pre-treatment & Post-treatment \\
\hline \multirow[t]{3}{*}{ Dye-based } & Acid & Silk, nylon, wool & Acid donor & Steam and wash \\
\hline & Reactive & Cotton, rayon & Alkali & $\begin{array}{l}\text { High temperature, } \\
\text { steam, and wash }\end{array}$ \\
\hline & Disperse & Polyester & Thickener & Steam and wash \\
\hline Pigment-based & & All & No & Dry heat \\
\hline
\end{tabular}

Source: $\mathrm{Fu}(2006)$

These challenges require both advances in scientific knowledge and practical solutions. Although pigment-based inks are available in the global market from Huntsman, DuPont, Irgaphor and some other chemical companies (Fang, 2006), their quality is still problematic and thus R\&D opportunities exist inside and outside of these giant companies. Opportunities for individuals and organisations to address these technological problems are expanded with the rise of nanotechnology material engineering capabilities in an increasing number of countries, including emerging economies. Nanotechnology not only provides tools for observation, examination and explanation at the atomic scale, but also allows the manipulation of nano-particles to develop materials with desired properties and functions. The focal technology of nano-pigment ink production offers an example of the application of nanotechnology to derive improved solutions for some long-standing technological problems in an existing industry.

\subsection{Research team, regional context, and the textile industry in China}

The research team studied in our case of nanomaterials innovation consists of a full professor, an associate professor, and several graduate students. The professor, Prof. Wu (pseudonym), led the entire development process, with other members entering and exiting at different points. Prof. Wu earned his doctoral degree from a Chinese textile university in the early 1990s. Within five years of completing his doctoral work, Prof. $\mathrm{Wu}$ had become well-recognised in the field of textile chemicals, printing, and finishing. He was awarded various honours and served as a senior member of leading national and international professional organisations including the Chinese Association of Textile Engineering, the Textile Engineering Education Committee of the Ministry of Education (MOE), and the American Association of Textile Chemists and Colorists (AATCC). The lead professor and the research team started their work on the focal technology at University M1 in Shandong province, China, before moving to University M2 in Jiangsu province, China.

Jiangsu and Shandong are two relatively well-developed provinces in China. Both of them are among the top three Chinese provinces by real GDP per capital (Government Online Resource, 2010). Jiangsu is north of the Shanghai metropolis and is in the Yangtze River Delta region, with a border to the Yellow Sea. Shandong is north of Jiangsu Province, also bordering the coast, in the Bohai Bay Region. Both regions contain concentrations of economic activities with high levels of entrepreneurial development (Alcorta et al., 2009; Kroll and Liefner, 2008). The two provinces (especially Jiangsu) have been large recipients of public science and technology investment and have relatively large numbers of $\mathrm{R} \& \mathrm{D}$ personnel. Using funding acknowledgements of nanotechnology publications, Wang and Shapira (2011) identify 


\section{Technology development trajectory}

As with most new technologies, the development of nano-pigment inks has been a continuous process, with subsequent improvements built upon earlier efforts and achievements. However, to structure the analysis, we consider the development of our focal technology case in three stages (see Figure 2). The nascent stage refers to the period from late 1999 to 2002. During this period, when Prof. Wu was at University M1, the basic idea was born, and the seed money for initial development was solicited from public research programmes. The period from 2003 to 2005 - the maturation technology stage - saw notable progress in research outcomes, including publications, patents, and samples from the lab as well as a pilot production line. The commercialisation stage began after government-sponsored projects had identified pathways for technology deployment. Since then, public support has faded away, while private sector engagement has grown. The following sections discuss these three stages in further detail.

\subsection{The nascent stage (1999-2001)}

Prof. Wu's ideas for engaging in producing inks for digital inkjet textile printing can be traced back to October 1999, when he attended the AATCC annual conference and exhibition (personal interview). The then latest achievements and problems facing the global community of textile dyeing and printing were presented at the conference and recorded, by Prof. $\mathrm{Wu}$, in an article published in China. The promising prospects for digital inkjet printing technology depicted at the conference, combined with the potential market size and environmental problems of China's textile industry, inspired and motivated Prof. Wu to initiate research on 'green' printing technology (personal interview).

The first move was to apply for research funding. Based on a preliminary literature review, a project was proposed to the National Science Foundation of China (NSFC). A grant of RMB 230,000 (about US\$ 32,900) was awarded in 2001. The NSFC grant was rather modest but it did provide seed support to develop a fundamental understanding of the properties of polycarboxylic molecules. The project led to multiple publications (seven domestic articles and one international paper). The project also allowed Prof. Wu to accumulate new knowledge spanning different technological fields related to the principles of digital inkjet printing.

\subsection{The maturation stage (2002-2005)}

After acquiring a certain level of relevant scientific knowledge, the research team faced an infrastructure problem. They could not produce nano-scale pigments without an advanced milling machine, nor could they observe or test the properties of those particles at that small scale without a potential analyser, surface tension metre, and other apparatus specifically designed for nano-scale observation and examination. Additionally, they needed a digital printer. Under Prof. Wu's leadership, the team thus sought financial support from various sources by proposing different research projects related to the core of nano-pigment ink production technology. This led to two project awards funded in 2002 by nationally prestigious S\&T programmes, namely the National High-Tech R\&D Programme (or 863 Programme) (RMB 1.8 million or US\$257,000) and the National Key Technology R\&D Programme (RMB 0.5 million or US\$71,000). During this phase, 
additional complementary funding from local government, University M2, and a collaborative industry partner was committed to match the funds provided by the national programmes. The 863 Programme required an industrial partner who also needed to provide some funding. As it turned out, this first corporate partner only invested 200,000 RMB (US\$28,600) before leaving at an early stage due to organisational changes within the enterprise.

By the time the funding from the two national programmes was actually received, Prof. Wu had been appointed to University M2 as a distinguished professor and moved from Shandong to Jiangsu. University M2 offered additional support to Prof. Wu including start-up research funding and lab space and enabled the establishment of a research centre to develop technologies for inkjet textile printing (personal interview). Advanced equipment, valued at around RMB 2 million (about US\$285,000), was purchased from international vendors for the centre. The most expensive and important equipment items are listed in Table 2. With the new equipment installed, the research team was able to rapidly move forward.

Table 2 Major laboratory equipment made available to the case study research team (2002-2005)

\begin{tabular}{lcc}
\hline Name & Type & Purchased from \\
\hline Zeta potential analyser & Nano-ZS90 & Malvern Co., UK \\
High-pressure micro jet-milling machine & M110EHI & Microfluidics Co., USA \\
Grinding machine & MINIZETA & NETZSCH, Germany \\
Benchtop spectrophotometer & Premier 8400 & X-Rite Co., USA \\
Surface tension metre & CAM200 & KSV Co., Finland \\
Viscometer & LVDV-11+ & Brookfield Co., USA \\
Dispersion machine & RW20N & IKA Co., Japan \\
\hline
\end{tabular}

Source: Internal documents, University M2

In this three-year period, the team worked out solutions for advancing the focal technology. These included optimising methods and processes for engineering nano-scale pigments, making a multi-functional agent for a stable dispersion system containing nano-pigments, preparing a nano-scale hydrophilic pigment dispersion system, and producing waterborne nano-scale carbon black dispersion. This phase also saw 90 publications (69 domestic and 21 international) and 24 patent applications - a stepwise increase in research outputs. Among the patent applications, seven were granted and two of these were licensed. A pilot production line was built to produce ink samples and products containing nano-pigments ('pink series') and to meet small orders. The line produced inks in six colours (yellow, magenta, light magenta, cyan, light cyan, and black) based on $50-150 \mathrm{~nm}$ pigment particles. These inks were tested using relevant international quality indicators, such as wash and crock resistance. The results were published with comparisons to other similar products available in the market. The pilot line was used not only to demonstrate the quality of the ink products but also to derive production data so as to gauge the investment required for scale-up. It was estimated that RMB 5 million (US $\$ 0.7$ million) would be the cost for building a line able to produce 200 tons annually. 


\subsection{The commercialisation stage (2006-2011)}

The Ministry of Science and Technology (MOST), the 863 Programme sponsor, evaluated the funded project at the end of 2005 . The peer review concluded that the nano-pigment ink series developed by the research team had reached an international advanced level. This review also signalled that the technology was ready for commercial use, so no further project support was available from the public sector (personal interview). The decision to commercialise the technology was now up to the research team. If they decided to go for commercialisation, the team would have to take the responsibility. In our case, the lead researcher and several team members chose to devote themselves to marketing their technology. They actively contacted different textile enterprises, gathered information about company needs, and continued to develop and customise the technology to meet these needs. Among many improvements, the team developed a solution for making inks suitable for a new generation of digital inkjet textile printers with much faster printing speeds (personal interview).

In 2008, Prof. Wu left University M2 and returned to University M1 as a faculty member while also becoming director of an $R \& D$ centre of a textile enterprise group. The rest of the research team stayed at University M2. By December 2009, two distinct models of technology commercialisation had emerged. The first was an independent development and commercialisation model in which Prof. Wu founded a start-up firm to produce nano-pigment inks. Prof. Wu decided to found this company because he was dissatisfied with the royalties proposed by existing incumbent companies. The royalties offered were much lower than his expectation (personal interview). The second model was to license the technology. By early 2012, patent grants for nano pigment technology were licensed to three companies. However, according to the lead researchers, it took a long time to identify appropriate licensees and many rounds of negotiations were necessary to reach agreements on royalties (personal interview). Thus, while licenses were eventually agreed, the complications and time involved in making such arrangements encouraged the pursuit of the start-up route, as shown in this case. Prof. Wu continues to undertake publicly-sponsored research on key scientific and technical problems related to this technology. This supports his ongoing efforts at commercialisation and licensing, as well as offering potential new opportunities for exploitation.

\section{Assessment}

The case described in this paper highlights the strategies pursued by the lead professor and his research team to develop and commercialise a new nanotechnology in China. Four broad themes emerge. First, Prof. Wu refined and leveraged his reputation as a 'star scientist'. He developed new research capabilities in an emerging field and promoted these publicly during his early research career. This led to further opportunities to gain access to information at the technology frontier, including international conference participation. Funds were secured from a range of funding agencies and collaborative relationships were initiated with industry. This process of accumulation of human and social capital was a key preparatory step essential for exploiting new research ideas and confronting the many barriers embedded in China's current innovation system 
Second, Prof. Wu was clearly interested in both advancing scientific research and exploiting technological and commercial opportunities. This combination is still relatively new among Chinese researchers. It is common in Chinese research organisations to find intense pressure to publish scientific research and a related underemphasis on research commercialisation. Our interviews confirmed the observations by $\mathrm{Wu}$ (2010) that most universities in China still hesitate to fully embrace the commercialisation mission despite increasing demands from the private sector for scientific expertise and the growing availability of industry funding. Yet, given this environment, it is a viable strategy for researchers to focus on topics where scientific and technological knowledge can be developed hand in hand. Indeed, it was the 'dual knowledge' nature of nano-pigment ink production technology that attracted Prof. Wu's involvement (personal interview).

Third, Prof. Wu and other team members actively embraced entrepreneurial activities. The research team established and maintained links with local industry and local government, as disclosed in interviews with researchers, textile company managers, local government executives, and an executive from a local textile industry consortium. The research team established relationships with key actors in the relevant innovation system, and these good relationships in turn benefited the researchers by bringing them timely information of market needs, funding sources, and potential collaborators for technology transfer. The research team's entrepreneurial commitment is seen in their stance towards intellectual property rights (IPR). Without any requirement or bonus for patenting in both universities, the researchers still took action to file patent applications, paying the application fees and annual fees by themselves. When asked why they did this, research team members highlighted the value of the improved IPR protection system in China, the role of patents in eliciting industry partners, and most interestingly, that they have learned strategic skills on how to disclose only part of the technology yet conceal its core elements.

Finally, the researchers pursued an interdisciplinary approach. Nanotechnology is not a unified technology with definite boundaries, but one spanning over many scientific and technological fields. The nano-pigment ink production technology in our case exemplifies the interdisciplinary nature of nanotechnology as it entails knowledge from chemical engineering, material science, electronic engineering, digital technology (hardware and software), and chromatology. An interdisciplinary approach proved to be essential in advancing nano-pigment ink production technology. This involved pursuing practical goals and maintaining a problem-solving orientation based on access to contextual information from industry and consideration of technological, economic and environmental issues. In our case, the advancement of the technology required the investment of time and effort in acquiring knowledge from multiple fields, some of which were initially unfamiliar to the researchers, such as electronic engineering and digital printer technology.

\section{Discussion and conclusions}

Our case offers a narrative about technology development and commercialisation of nano-materials in China. Barriers and gaps, however, exist in the story partly as characteristics of Chinese innovation system and partly related to the nature of nanotechnology. Even though considerable funding and infrastructural facilities have 
been invested to promote the flow of university-based research to industry, innovation and technology transfer activities have not yet been fully valued and supported by Chinese universities (Wu, 2010; Wu and Zhou, 2011). In our case, this was reflected in the intense pressure (reported in personal interviews) to publish and the lack of organised technology transfer units to initiate and facilitate patenting, licensing, and other commercial processes. Universities in China have historically been regarded as institutions for educating knowledge workers and undertaking basic research but not for knowledge diffusion and application (Wu and Zhou, 2011). The competing requirements of education and research now present major challenges for academic scientists to commercialise their research. Even in our case, where new scientific knowledge was expected to be acquired through the technological development process, tensions between knowledge generation and commercialisation were reported by the researchers we interviewed. It forced these scientists to increase the number of hours worked each week. While this works for scientists with a strong entrepreneurial capability and propensity, the overriding pressure in China to publish may constrain many other scientists, particularly younger ones, from commercialising their scientific discoveries.

Our case illustrates a gap between private enterprises and researchers with regard to a new technology's potential value. In China, as elsewhere, enterprises (especially small and medium sized enterprises) are often risk-averse when considering new technologies due to limitations in financial and technology absorptive capabilities. They typically consider a new technology only if assured that the benefits clearly outweigh the costs. The information and experience needed to assess the benefits and tradeoffs associated with a new technology are not readily available. Most enterprises are thus reluctant to assume the risks of investing in and adopting an untested new technology, notwithstanding its potential to bring significant benefits to first movers in the market. This gap has been confirmed in a study analysing survey data from 950 industrial enterprises in Beijing, where these companies perceive that technologies developed from academic research are often immature and require costly further development with uncertain market value (Guan et al., 2005). Other studies of university-industry linkage in China also point to the discrepancy between researchers and industrial enterprises on the economic value of new university-developed technologies (e.g., Wu and Zhou, 2011; Zhou, 2005). On the other side, researchers frequently highlight the path-breaking significance and market potential of the new technologies that they develop. This leads some (although not the majority) of researchers to become more engaged in applied development and commercialisation. Yet, although equipped with scientific and technological knowledge, researcher's efforts to foster commercialisation can be stymied by the realities of market conditions including the reluctance or inability of enterprises and other investors to embrace new research innovations. Our case demonstrates these tensions and indicates how motivated researchers seek to find pathways through commercialisation barriers.

While challenges of research commercialisation are ubiquitous in China's innovation system, the novel and interdisciplinary nature of nanotechnology (and other emerging technologies) adds further complexities, as suggested in our case. The development of applications for a new technology is an iterative learning process for researchers, in which they test different approaches and, in an interdisciplinary domain, incorporate contextual information and knowledge from multiple fields. Enterprises will frequently have difficulties absorbing these new approaches into their frameworks of innovation and operation if they are simply assigned a receiving 'end of pipeline' role. Yet we do see 
significant changes in the innovation system in China which lean more towards concurrent and interactive formulations. Indeed, in our case, it was these changes that offered opportunities for the lead professor to overcome various structural barriers and navigate this particular nanotechnology development into the commercial sphere. One of the notable changes is the availability of more diversified funding sources. Funding agencies and programmes in China now support both technological commercialisation and scientific advancement, with increased industry funding also flowing into academic research via various avenues (consulting, licensing, cooperative commercialisation, etc.). While the higher education sector in China has long operated on the state-centred model (Wu and Zhou, 2005), such changes allow faculty researchers with entrepreneurial ambitions to have greater autonomy and flexibility to choose topics and ways to interact with industry. Our case study suggests that these diverse relationships, backed by financial support, whether vertically or horizontally, enhance the dynamics of interactions and learning and especially benefit technologies that are still in their initial development stage.

Another change is the greater recognition of, and more effective protection for, IPR. Although patent licensing serves as a major mechanism for universities to diffuse innovation in the West, it has been underutilised in China (Wu and Zhou, 2011). There are both institutional level and individual level reasons. Historically, Chinese patent procedures for intellectual property protection were designed not so much for creating commercial opportunities, but rather for evaluating research quality (personal interview with SIPO official). Patent applicants and review and regulatory agencies (including SIPO) typically put more emphasis on the quality signaled by patents than on measures to protect the intellectual property itself. At the individual level, faculty entrepreneurs prefer consultancy or other informal commercial activities to patent licensing because the latter option implies royalty sharing with the university or department as well as transaction and other costs associated with formal IPR methods (Wu and Zhou, 2011). However, in recent years, SIPO has sought to adapt the Chinese IPR system to better support and protect formal technology transfer mechanisms. Enterprises and researchers increasingly recognise the importance of IPR and are aware of the improving IPR environment in China. This makes them more appreciative of the need to strategically acquire and protect intellectual property. Further training, support and regulatory enforcement is surely necessary, but - as our case demonstrates - intellectual property is now becoming increasingly important in the commercialisation process of scientific discovery in China, alongside other efforts to stimulate innovation. Nanotechnology, as a cutting-edge technology, could be among the beneficiaries of the changing IPR context in China. Potentially, this could accelerate advanced technology transfer from Chinese universities to enterprises engaged in Chinese and global markets.

Our analysis of a case of innovation in nano-pigment ink technology provides a snapshot of the Chinese innovation system which emphasises learning, change, and the development of a more dynamic environment. It also underscores two arguments at the core of the notion of national innovation systems (Freeman, 1995; Lundvall, 1992; Nelson, 1992). First, universities have the potential to play a crucial role in an advancing knowledge economies and spillovers. Second, processes of learning and interaction are fundamental building blocks for an effective innovation system. To return to the original question raised at the beginning of this study, our case study suggests that the development trajectory of nanotechnology in China demonstrates both the science-based 
nature of nanotechnology and the influence of the Chinese innovation context. Our case highlights the changing role of universities, the emergence of entrepreneurial academic research leaders, the availability of more diverse forms of sponsorship to support new technology commercialisation, and a more favourable IPR regime for emerging technologies. Meanwhile, the limitations of this case should be recognised: although we were able to discern the development of new approaches to research commercialisation in an emerging technology, we cannot in this single study quantify or generalise the extent of such developments nor assess the overall business and technological implications. What our study does offer is a signal that shifts are underway in the Chinese innovation system, with capabilities being built not only to undertake advanced research in emerging technologies but also to engage research institutions in the entrepreneurial application and deployment of that new scientific knowledge into industry.

\section{Acknowledgements}

The authors thank the Gore Innovation Project, Chemical Heritage Foundation (CHF) for sponsorship of this research. Any findings and conclusions are those of the authors and do not necessarily reflect the views of the CHF.

\section{References}

Alcorta, L., Tomlinson, M. and Liang, A.T. (2009) 'Knowledge generation and innovation in manufacturing firms in China', Industry and Innovation, Vol. 16, Nos. 4-5, pp.435-461.

Babbie, E. (2000) The Practice of Social Research, Wadsworth/Thomson Learning, Belmont, CA.

Cahill, V. (2006) 'The evolution and progression of digital printing of textiles', in Ujiie, H. (Ed.): Digital Printing of Textiles, CRC Press, Boca Raton.

Fang, K.J. (2006) 'Digital inkjet printing technology', Dyeing and Finishing, Vol. 32, No. 18, pp.44-46.

Fang, K.J., Fu, S.H., Zhang, X., Tian, A.L. and Wang, C.X. (2004) 'Development of digital inkjet printing', Dyeing and Finishing, Vol. 30, No. 24, pp.48-51.

Freeman, C. (1995) 'The national innovation systems in historical perspective', Cambridge Journal of Economics, Vol. 19, No. 1, pp.5-24.

$\mathrm{Fu}$, Z. (2006) 'Pigmented ink formulation', in Ujiie, H. (Ed.): Digital Printing of Textiles, CRC Press, Boca Raton.

GAO (1990) Case Study Evaluations, Program Evaluation and Methodology Division, US General Accounting Office, Washington, DC.

Government Online Resource (2010) The Economy of Guangdong, Jiangsu, and Shandong Accounts for One Third of China's GDP in 2009 [online] (accessed 20 March 2010).

Guan, J., Yam, R. and Mok, C. (2005) 'Collaboration between industry and research institutes/universities on industrial innovation in Beijing', Technology Analysis \& Strategic Management, Vol. 17, No. 3, pp.339-353.

Kroll, H. and Liefner, I. (2008) 'Spin-off enterprises as a means of technology commercialisation in a transforming economy - evidence from three universities in China', Technovation, Vol. 28, No. 5, pp.298-313.

Lau, C.K., To, K.M., Zhang, Z.M. and Chen, J. (2009) 'Determinants of competitiveness: observations in China's textile and apparel industries', China \& World Economy, Vol. 17, No. 2, pp.45-64. 
Li \& Fung Research Center (2006) Textile and Apparel Clusters in China, Industrial Cluster Series, Hong Kong [online] http://www.idsgroup.com/profile/pdf/industry_series/LFIndustrial5.pdf (accessed 2 January 2011).

Liu, L.W. and Zhang, L.D. (2005) 'Nanotechnology in China - now and in the future', Nanotechnology Law \& Business, Vol. 2, No. 4, pp.397-403.

Lundvall, B.A. (Ed.) (1992) National Systems of Innovation: towards a theory of Innovation and Interactive Learning, St. Martin's Press, London \& New York.

Mowery, D.C. (2011) 'Nanotechnology and the US national innovation system: continuity and change', Journal of Technology Transfer, Vol. 36, No. 6, pp.697-711.

Nelson, R.R. (Ed.) (1992) National Systems of Innovation: A Comparative Study, Oxford University Press, Oxford.

Ohno, A. (2006) 'Inkjet textile printing: keys for success to be the real industry', The 29th Global Ink Jet and Thermal Printing Conference, Konica Minolta, Amsterdam, Holland.

Shapira, P. and Wang, J. (2009) 'From lab to market? Strategies and issues in the commercialization of nanotechnology in China', Asian Business \& Management, Vol. 8, No. 8, pp.461-489.

Shepard, J. and Greene, R.W. (2003) Sociology and You, Glencoe McGraw-Hill, Ohio.

Tang, L. and Shapira, P. (2011) 'China-US scientific collaboration in nanotechnology: patterns and dynamics', Scientometrics, Vol. 88, No. 1, pp.1-16.

Wang, J. and Shapira, P. (2011) 'Funding acknowledgement analysis - an enhanced tool to investigate research sponsorship impacts: the case of nanotechnology', Scientometrics, Vol. 87, No. 3, pp.563-586.

Wu, W.P. (2010) 'Managing and incentivizing research commercialization in Chinese universities', Journal of Technology Transfer, Vol. 35, No. 2, pp.203-224.

$\mathrm{Wu}$, W.P. and Zhou, Y. (2011) 'The third mission stalled? Universities in China's technological progress', Journal of Technology Transfer, No. 6, online first, DOI: 10.1007/s10961-0119233-8.

Yin, R.K. (2009) Case Study Research: Design and Methods, 4th ed., SAGE Publications, California.

Youtie, J., Shapira, P. and Porter, A.L. (2008) 'Nanotechnology publications and citations by leading countries and blocs', Journal of Nanoparticle Research, Vol. 10, No. 6, pp.981-986.

Zhou and Leysdorff (2006) 'The emergence of China as a leading nation in science', Research Policy, Vol. 35, No. 1, pp.83-104.

Zhou, Y. (2005) 'The making of an innovative region from a centrally planned economy: institutional evolution in Zhongguancun science park in Beijing', Environment and Planning $A$, Vol. 37, No. 6, pp.1113-1134. 